\title{
Mental Toughness in Talented Youth Tennis Players: A Comparison Between on-Court Observations and a Self-Reported Measure
}

\author{
by \\ Ruben Houwer1, Tamara Kramer1,2, Ruud den Hartigh ${ }^{3}$, Nikki Kolman', \\ Marije Elferink-Gemser ${ }^{1}$, Barbara Huijgen ${ }^{1}$
}

\begin{abstract}
In tennis, mental toughness is often considered highly important in achieving the elite level. The current study is the first to examine behavioural expressions of mental toughness on the court and their relationships with selfreported measures. Based on the input of five experienced tennis coaches of junior tennis players and behaviours used in previous studies, we developed a taxonomy consisting of six positive behaviours and ten negative ones. To investigate the relationship between these on-court behaviours of mental toughness and how the players rated their own mental toughness, emotional control in particular, six talented tennis players (aged 10-13) were recorded during tennis matches and filled out the self-reported measure of mental toughness (MTQ48). The intra- and inter-rater reliability of the taxonomy was high. With regard to the relationships between on-court behaviours and self-reported mental toughness (total score and subscale emotional control), results revealed no significant correlations between the ratios of positive and negative behaviours (range $r=-0.49-0.11, p>0.05)$ or between the variability of negative behaviours $(r=$ $0.54 \& r=0.10, p>0.05$ ) and the self-reported measure. However, interestingly, we found negative correlations between the variability of positive behaviours and self-reported mental toughness $(r=-0.93 \mathcal{E} r=-0.84, p<0.05)$. These results indicate that variability in on-court behaviours provides interesting information about tennis players' mental toughness, more specifically on the (in)stability of their psychological state during a match.
\end{abstract}

Key words: emotional control, behavioural expressions, variability, performance, youth sports.

\section{Introduction}

"In these particular matches and circumstances, mental strength probably plays the most important role. In winning those matches, you need to be able to find that inner strength, mental, physical, emotional, especially when you're down in the finals and when you're playing a top rival" (Novak Djokovic, World's number 1 in men tennis (May 2016), after winning the final of the Australian Open 2015).

In tennis, the psychological aspects are considered to be very important, especially at the higher performance level (Weinberg, 1988). In the past years, more and more research has demonstrated that psychological skills play a crucial role in becoming an elite athlete (ElferinkGemser et al., 2004; van Yperen, 2009). One of the key psychological skills is mental toughness, which has been studied in different kinds of

1 - Center for Human Movement Sciences, University Medical Center Groningen, University of Groningen, the Netherlands.

2 - HAN Sports and Exercise, HAN University of Applied Sciences, the Netherlands.

3- Department of Psychology, Faculty of Behavioural and Social Sciences, University of Groningen, the Netherlands. 
sports (Bull et al., 2005; Gucciardi et al., 2008; Jones et al., 2002, 2007; Weinberg et al., 2009). Jones et al. $(2002,2007)$ conducted two studies in an attempt to provide a coherent definition of mental toughness. Based on reports of elite performers, their coaches and sport psychologists, the authors defined mental toughness as having the natural or developed psychological edge that enabled an athlete to be more consistent and better than opponents in remaining determined, focused, confident and in control of thoughts and feelings.

A component of mental toughness that is particularly relevant in sports, especially tennis, is the control of thoughts and feelings (i.e., emotional control, see Clough et al., 2002; Gould et al., 1999; Gucciardi et al., 2008; Jones et al., 2007). For instance, Loehr (1986) identified positive energy (i.e. the ability to become energized from positivity) and negative energy control (i.e. handling negative emotions) as attributes of mental toughness. In addition, research showed that positive/negative affectivity (i.e. the experience of positive and negative feelings or emotions) is a key correlate of mental toughness (Sheard and Golby, 2006a, 2006b). Clough and Strycharczyk (2012) stated that mental toughness could be predicted by negative behaviours. Thus, the degree to which positive and negative emotions are experienced and behaviours are expressed is an important aspect of mental toughness. In addition, athletes with high levels of emotional control are "able to keep anxieties in check and are less likely to reveal their emotional state to other people" (Clough and Strycharczyk, 2012). The latter suggests that the athletes' state should be relatively stable, more specifically mentally tough behaviour is characterized by low variability in positive or negative behaviours.

Most measurements of mental toughness involve self-reported measures. In general, this method is least time-consuming and simple to administer (Paulhus and Vazire, 2007). The most widely-used self-reported measure is the MTQ48, which departs from the 4C model of mental toughness (Clough et al., 2002). This model, developed by athletes, coaches and sport psychologists, identified four components of mental toughness: commitment, challenge, confidence and control. The last two scales are divided into two subscales, confidence in abilities and interpersonal confidence, and life control and emotional control, respectively. Research using the MTQ48 showed that higher levels of mental toughness were related to the experience of less stress and more control (Kaiseler et al., 2009). However, the use of only questionnaires has some disadvantages. In questionnaires, there is the likelihood of socially desirable responding and they do not measure actual behaviour on the court, the place where mental toughness is demonstrated (or not). Therefore, multiple studies recommend multi-source measures of mental toughness, with self-reported questionnaires as well as reports by others (e.g. coaches), performance outcomes and observations (Crust, 2008; Middleton et al., 2004). Until now, no study has used multiple methods for measuring mental toughness. To bridge the gap between actual oncourt behaviour and self-reported measures of mental toughness, it would be interesting to apply both kinds of methods.

In order to measure on-court behaviour, a taxonomy should be developed. Research suggests that elite coaches and athletes could play an important role in identifying positive and negative on-court behaviours in tennis and soccer (Diment, 2014; Hanegby and Tenenbaum, 2001; van Raalte et al., 1994). Hanegby and Tenenbaum (2001) conducted an observational study of negative mental behaviours in junior elite tennis players. For the observation, a taxonomy of negative mental behaviours was developed with the help of highly ranked tennis players. However, this study lacked the positive mental behaviours during a tennis match. Given that (a) positive behaviours should be taken into account to reveal actual positive expressions of mental toughness, and (b) positive behaviours are observable in tennis matches (e.g. making a fist, positive self-talk), a taxonomy of mentally tough behaviour in tennis should take into account positive as well as negative behaviours.

In the current study, we aimed to examine tennis players' behavioural expressions of mental toughness on the court, and their relationships with self-reported measures. The first step was to develop a taxonomy of positive and negative behaviours of mental toughness for on-court observations of junior tennis players. Second, we investigated the relationship between 
observations of mental toughness and a selfreported measure of mental toughness, in particular emotional control. This may provide new insights into how positive and negative behaviours as well as emotional on-court control are related to the self-reported mental toughness. Given the fact that the amount of positivity and negativity as well as the variability of these positive and negative behaviours seem to be relevant factors of mental toughness, the focus was laid on these specific aspects. However, no clear hypothesis was presented, considering that this study was the first one to investigate mental toughness in this manner.

\section{Material and Methods}

\section{Participants}

Six male tennis players, aged 10-13 (Mage $=$ $11.8 \pm 1.3$ years), were approached to participate in the study. On average, they started to play tennis at the age of $5.8 \pm 1.5$ years and the players were ranked $28^{\text {th }}, 44^{\text {th }}, 75^{\text {th }}, 94^{\text {th }}, 102^{\text {th }}$ and $138^{\text {th }}$ at the national youth ranking list in the Netherlands. The players were trained at a regional tennis academy in the north of the Netherlands and had on average $12.5 \pm 2.8$ hours of training per week, with one hour per week of mental training.

\section{Measures of observations}

\section{Development of the taxonomy}

Five coaches of youth tennis players assisted in the development of a taxonomy of mentally tough behaviours in tennis as a guideline for the observations. On average, these coaches had $27 \pm 2.1$ years of experience and they were working 20 hours per week with young talented tennis players. First, the coaches were asked to compose a list of ten positive and ten negative behaviours that could occur on the court during a match. The positive behaviours needed to indicate a high level of mental toughness, while the negative behaviours needed to indicate a low level of mental toughness. Another condition was that the behaviours had to be observable. Second, the lists of the coaches were compiled so that there was one list of positive and one list of negative behaviours (50 behaviours each). There was some overlap between the coaches regarding the positive and negative behaviours they mentioned. These overlapping behaviours were analysed in collaboration with three researchers having played at national level and afterwards, the list consisted of 26 unique positive and 26 unique negative behaviours. Third, the adjusted lists were sent back to the coaches. At that time, they were asked to rank the ten most important positive and negative behaviours (from 1 to 10) indicative of mental toughness. Finally, it was discussed by the same three researchers whether the behaviours were easily observable. The final version of the taxonomy (Table 1) consisted of the most important observable behaviours of mental toughness mentioned by tennis coaches, supplemented with observable behaviours from previous literature (Hanegby and Tenenbaum, 2001; van Raalte et al., 1994). Based on the taxonomy, a coding book (the coding book could be requested by contacting the corresponding author) was created. It contained information about how the taxonomy was developed, an overview of the behaviours and which coding character belongs to which behaviour.

Reliability of the taxonomy

The inter-rater reliability of the taxonomy was determined by observation of one match by the first and second observer, who both had undergone brief training to use the coding system. In addition, the intra-rater reliability was assessed by observing one match twice by the same observer. Also, Cohen's kappa ( $\kappa)$ was calculated to correct for chance. When an intra- and interobserver agreement of $80 \%$ was achieved, it could be concluded that the taxonomy was a reliable measurement tool for the observations (McHugh, 2012).

\section{Ratio of positive/negative behaviours}

The ratio of positive/negative behaviours was calculated using the first and the second set of the three set matches, to include both a winning and a losing set. First, a positive and a negative ratio were calculated for both sets. The positive ratio was calculated by dividing the amount of positive behaviours by the amount of points won. The negative ratio was calculated by dividing the amount of negative behaviours by the amount of points lost. Second, the main ratio was calculated for both sets by dividing the positive ratio by the negative one. This represented the ratio between the amount of positive and negative behaviours for each set. The higher this ratio, the more positive behaviours were executed compared to the negative ones. At last, the average of both sets was calculated. This produced three ratios in total: 
the main ratio of the winning set, the main ratio of the losing set and the average of both ratios.

Variability

To analyse the variability during a match, the first and the second set of the three set matches were split into blocks of 4 or 5 games. For each block, the same ratios as described above were calculated: one ratio for the positive behaviours and one ratio for the negative behaviours. The variability per block was calculated by totalling the differences in ratios between two consecutive blocks and dividing the total variability by the amount of blocks minus one. This was done for the positive and the negative behaviours separately. The lower the ratio, the lower the variability during the first two sets.

\section{Measure of self-reported mental toughness}

Mental Toughness Questionnaire 48 items (MTQ48)

A translated version of the MTQ48 was used. The MTQ48 (Clough et al., 2002) is a 48-item self-reported questionnaire using a 5-point Likert scale ranging from 1 (strongly disagree) to 5 (strongly agree) which measures four scales of mental toughness (the $4 \mathrm{C}^{\prime} \mathrm{s}$ ): challenge (8 items), commitment (11 items), control (14 items) and confidence (15 items). The control scale consists of two subscales: emotional control (7 items) and life control (7 items). Higher scores on the scales indicated higher levels of mental toughness. The average score on the subscale of emotional control as well as the total average score on the MTQ48 were used for the analysis. The original version repeatedly showed sufficient internal consistency on all subscales and on the whole scale $(0.70-$ 0.91), overall high test-retest coefficients (0.80 0.87 ) and good factorial validity (Strycharczyk and Clough, 2014).

\section{Procedure}

Fourteen matches at five national junior ranking tournaments played in the Netherlands were observed using the developed taxonomy of mentally tough behaviours. The recorded players and their parents received an information letter and gave informed consent. The opponents during the matches and their parents gave verbal permission for the observation of the other player. Ethical approval was received from the ethics committee of the Center for Human Movement Sciences, University of Groningen (The Netherlands) and the study was consistent with the ethical requirements for human experimentation in accordance with the Declaration of Helsinki. The matches were recorded with two high definition cameras placed outside the court and the fence, one aimed at one half of the court, the other one at the other half. Only one player per match was recorded, therefore, it was possible to rotate the camera to follow the player during the point. When the players changed sides, the other camera was used to record the player. During the match the score was kept with the app Tennis Math (SUPREMATIC, 2009) on a tablet, including service tracking and point results. The recorded matches were observed and coded using Media Coder 2010 (Bos and Steenbeek, 2006). Table 1 shows how the taxonomy was included in the program (translated to English).

Twelve weeks after the recordings of the tennis matches, participants filled out a questionnaire about mental toughness. In addition, participants responded to some general questions about the amount of training hours per week, types of training and the age when they started to play tennis. The players took the questionnaire on paper accompanied by one test leader.

\section{Statistical Analysis}

First of all, all fourteen matches were observed and coded. Only the three-set matches were used for the analysis, for two reasons. First, this way we only examined the matches in which the level of the participant was relatively equal to the opponent (e.g., it would be less informative to analyse a match in which the participant was much better or worse than the opponent). Second, by looking at the first two sets it was possible to make the comparison between a losing set and a winning set. All players except one played a three-set match. Therefore, this one player (ranked $75^{\text {th }}$ ) was not taken into account for further analyses.

IBM SPSS Statistics 23 (IBM Corp. Somers, NY) was used for the statistical analyses of the observations and questionnaires. To assess the relationship between scores on the observations and the self-reported measure of mental toughness, the scores on emotional control as well as the total mental toughness score of the MTQ48 were used for comparison with the variables of the observations. A Pearson correlation $(r)$ was 
used to assess:

1) The correlation between the main ratio positive/negative behaviours and the average score of the MTQ-48

2) The correlation between the main ratio positive/negative behaviours and the average score of the MTQ-48, subscale of emotional control

3) The correlation between the variability of the positive and negative behaviours and the average score of the MTQ-48

4) The correlation between the variability of the positive and negative behaviours and the average score of the MTQ-48, subscale of emotional control

Guidelines of Cohen (1988) were followed, thus, where an $r$ between 0.1 and 0.3 indicated a weak correlation, a value between 0.3 and 0.5 meant a moderate correlation and an $r$ higher than 0.5 indicated a strong correlation. The level of significance was set at $p$ (one-tailed $)=0.05$.

\section{Results}

Taxonomy (For an overview of behavioural frequencies of the on-court observations, contact the corresponding author)
In total, six positive behaviours and ten negative behaviours were included in the final taxonomy, divided into different categories as displayed in Figures 1 and 2. The behaviours that were added from previous research studies are marked with an asterisk in the figures. The interrater reliability of the taxonomy was $\kappa=0.77$ (agreement $81 \%$ ) and the intra-rater reliability was $\kappa=0.92$ (agreement 94\%). This means substantial inter-rater reliability and almost perfect intra-rater reliability.

\section{On-court observations and MTQ48}

The results of the scores on the MTQ48 and the observational variables are presented in Table 2. First, the table shows that many differences exist between the players regarding their ratio of positive and negative behaviours. Second, the averages of the ratio of positive behaviours show that players were almost equally positive in both sets (0.66 and 0.64). However, the negative ratios show that players were more negative during a losing set than during a winning set (0.66 and 0.47). This is also apparent in the individual ratios, where all players but one were more negative in the losing than in the winning set.

\section{Table 1}

Taxonomy of positive and negative behaviours included in the coding program

\begin{tabular}{ll}
\hline - & $\mathrm{K}=$ Cheer (e.g. "come-on" or "yes") \\
- & $\mathrm{I}=$ Instructive self-talk \\
- & $\mathrm{P}=$ Positive self-talk \\
- & $\mathrm{C}=$ Complimenting opponent \\
- & $\mathrm{O}=$ Fist bump \\
- & $\mathrm{F}=$ Exprticing the stroke motion \\
- & $\mathrm{N}=$ Negative self-talk \\
- & $\mathrm{T}=$ Expression of disappointment \\
- & $\mathrm{X}=$ Anger or frustration about external factors (opponent, court, weather) \\
- & $\mathrm{E}=$ Disappointment about external factors (opponent, court, weather) \\
- & $\mathrm{H}=$ Negative attitude (gestures with arms or the head, trudging on the court) \\
- & $\mathrm{Z}=$ Complaining non-verbally about external factors (opponent, court, weather) \\
- & $\mathrm{M}=$ Disappointment aimed at external things (racket, net, fence, court or chair) \\
- & $\mathrm{Y}=$ Other verbal behaviours \\
\hline
\end{tabular}


Table 2

Results of the self-reported measures and observational variables ( $W=$ set won, $L=$ set lost, $E C=$ emotional control, pos. $=$ positive, ned. $=$ negative)

\begin{tabular}{|c|c|c|c|c|c|c|}
\hline & Player 1 & Player 2 & Player 3 & Player 4 & Player 5 & Average (SD) \\
\hline MTQ48 total & 4.25 & 3.80 & 3.44 & 3.50 & 3.38 & $3.67(0.32)$ \\
\hline MTQ48 EC & 4.14 & 4.14 & 3.14 & 3.00 & 2.57 & $3.40(0.63)$ \\
\hline Ratio positive $\mathrm{W}$ & 0.52 & 0.34 & 0.75 & 1.16 & 0.42 & $0.64(0.30)$ \\
\hline Ratio negative $\mathrm{W}$ & 0.79 & 0.15 & 0.18 & 0.60 & 0.62 & $0.47(0.26)$ \\
\hline Ratio pos/neg W & 0.66 & 2.27 & 4.17 & 1.93 & 0.68 & $1.94(1.29)$ \\
\hline Ratio positive L & 0.50 & 0.42 & 0.82 & 1.31 & 0.27 & $0.66(0.37)$ \\
\hline Ratio negative L & 0.86 & 0.23 & 0.64 & 0.54 & 1.03 & $0.66(0.27)$ \\
\hline Ratio pos/neg L & 0.58 & 1.83 & 1.28 & 2.43 & 0.26 & $1.28(0.79)$ \\
\hline $\begin{array}{l}\text { Average ratio } \\
\text { pos/neg W/L }\end{array}$ & 0.62 & 2.05 & 2.73 & 2.18 & 0.47 & $1.61(1.01)$ \\
\hline Variability pos. & 0.21 & 0.27 & 0.33 & 0.28 & 0.35 & $0.29(0.05)$ \\
\hline Variability neg. & 0.55 & 0.19 & 0.34 & 0.34 & 0.35 & $0.35(0.11)$ \\
\hline
\end{tabular}

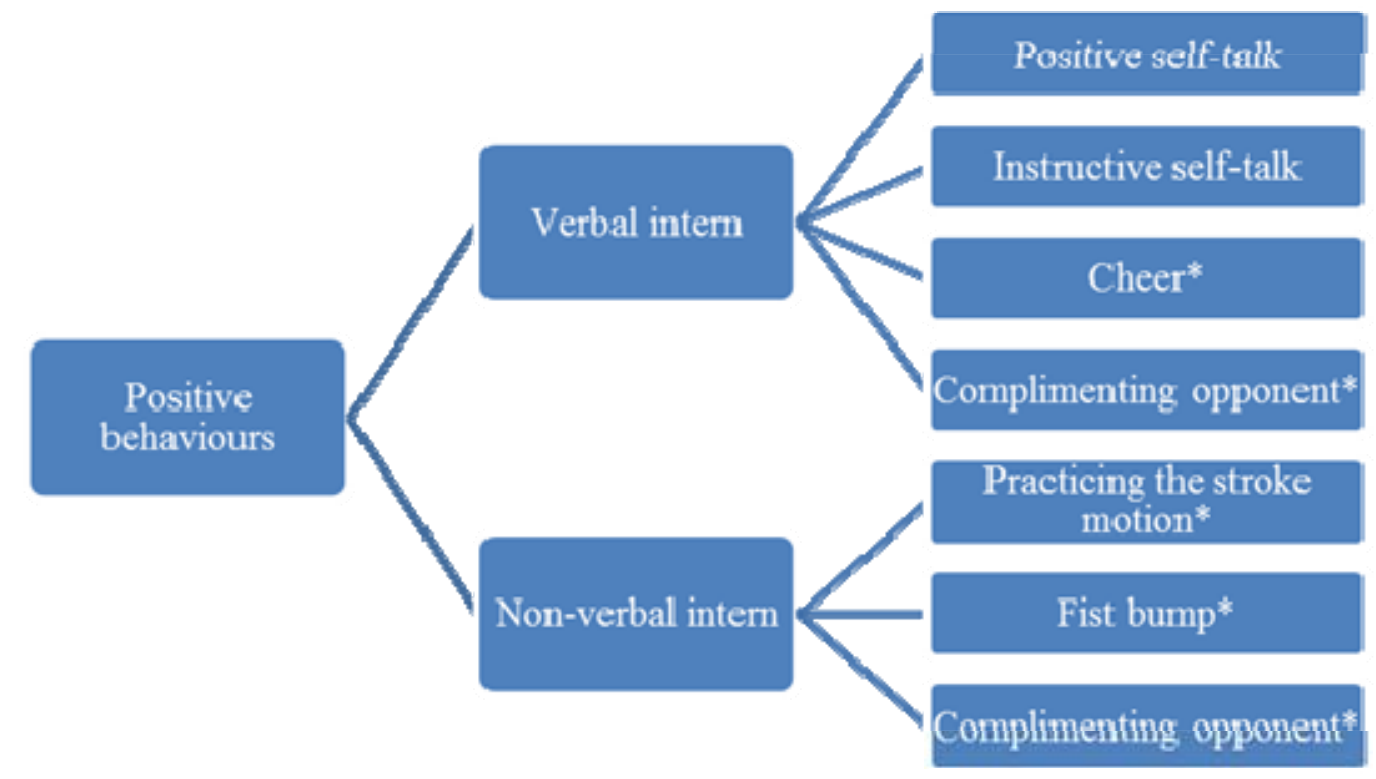

Figure 1

Positive behaviours of mental toughness divided into two categories $(*=$ behaviour added from literature: Hanegby and Tenenbaum, 2001; van Raalte et al., 1994).) 


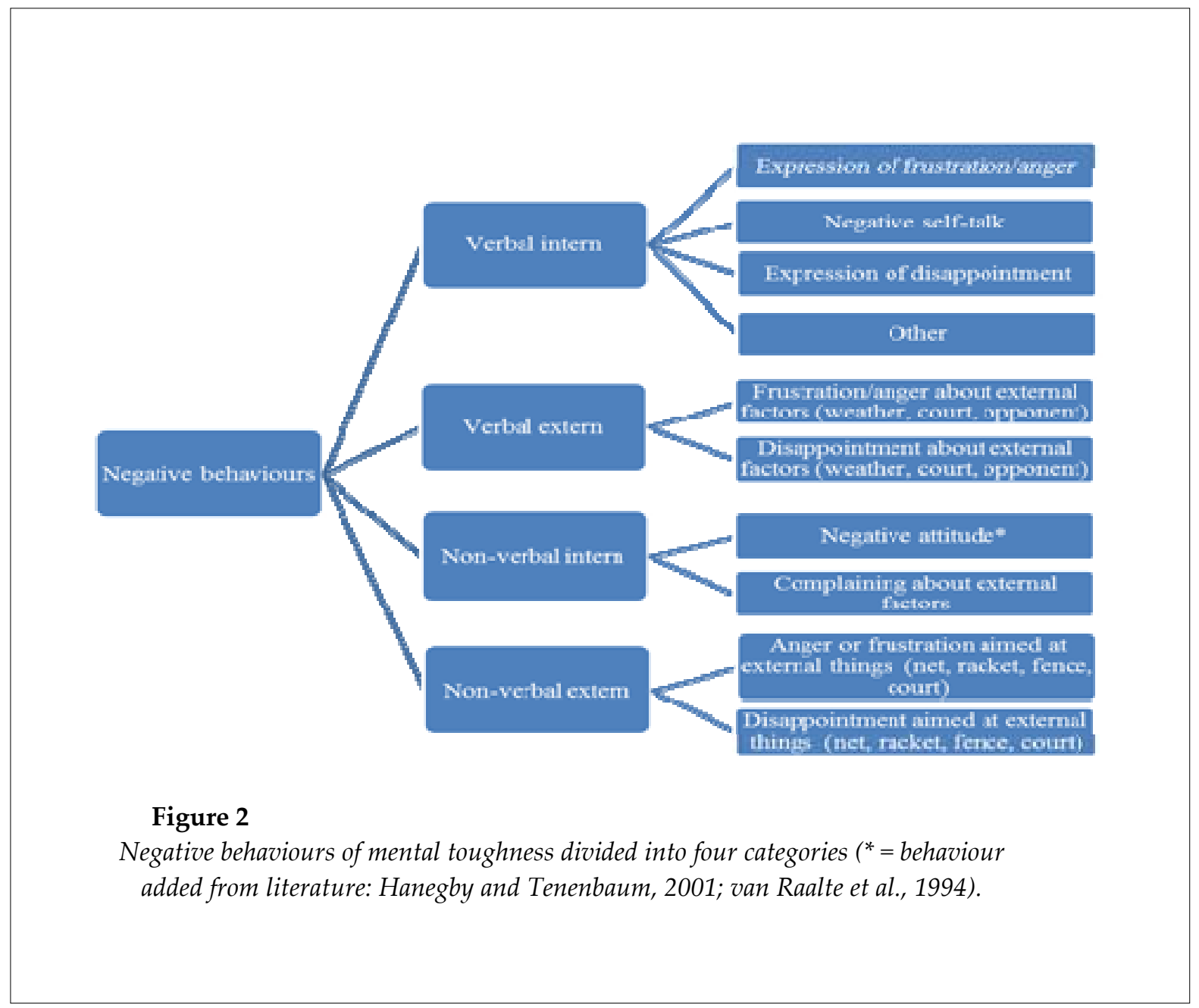

Pearson correlation showed no significant relationships between scores on the MTQ48 (total score and subscale of emotional control) and the ratios of positive and negative behaviours (range $r$ $=-0.49-0.11, p>0.05)$. Furthermore, no correlations were found between the MTQ48 (total score and subscale of emotional control) and the variability of negative behaviours $(r=0.54 \& r$ $=0.10, p>0.05)$. However, a strong significant negative correlation was found between the total MTQ48 score and variability of positive behaviours $(r=-0.93, p<0.05)$. Additionally, a strong significant negative correlation was found between the MTQ48 score on emotional control and the variability of positive behaviours $(r=-$ $0.84, p<0.05)$.

\section{Discussion}

In the current study, we aimed to examine tennis players' behavioural expressions of mental toughness on the court, and their relationships with self-reported measures. For this aim, we first developed a taxonomy of positive and negative behaviours of mental toughness for on-court observations of junior tennis players. The total taxonomy consisted of six positive and ten negative behaviours, and was composed on the basis of interviews with coaches of young talented tennis players and existing behaviours already described in the literature. Second, we investigated the relationship between the observations of mental toughness and selfreported mental toughness.

The taxonomy was considered to be a reliable instrument in coding the positive and negative on-court behaviours. No significant relationships were found between the ratios of positive and negative behaviours, and the selfreported mental toughness. Furthermore, no correlations were found between the variability of 
the negative behaviours and the self-reported mental toughness. The fact that no correlations were found between the ratios of positive and negative behaviours and the self-reported mental toughness suggests that the amount of positivity and negativity showed on the court was not related to the scores on the self-reported measure of mental toughness. This is in line with an earlier study of Crust (2009) who investigated the relationship between mental toughness and the experience of more or less intense emotions. No relationships were found between the MTQ48 and the Affect Intensity Measure. This means that there is no evidence to suggest that mentally tough players are less emotional on the court.

However, a strong negative relationship was found between the variability of positive behaviours and scores on the self-reported mental toughness. More specifically, players with higher variability of the positive behaviours during the match showed lower scores on the self-reported mental toughness. This is in line with the assumption that mentally tough players need to control their emotions during competition and thus, do not show high variability in emotions during a tennis match. Players with higher levels of mental toughness might be more capable of controlling their emotions during performance. In line with this idea, research has shown that emotional intelligence (i.e. managing and controlling emotions) is related to mental toughness (Nicholls et al., 2015). However, we did not find a significant relationship between selfreported mental toughness and variability in negative behaviours. Still, we should note that the correlation was relatively high and therefore, not meaningless. Further research should elaborate the variability of these behaviours of mental toughness to gain more information about the relationship between the positive and negative variability, and self-reported measures of mental toughness.

One of the major strong points of the study is that actual on-court behaviour was measured as a part of multi-source measures, i.e. self-reported measures as well as on-court observations, of mental toughness. After all, to understand the extent to which athletes are sensitive to positive and negative events during a match, their behavioural (and psychological) responses should also be examined during the match (Den Hartigh et al., 2014, 2016). Another strong point is that the taxonomy was developed with the help of several elite coaches of junior tennis players, supplemented by behaviours used in earlier studies (Hanegby and Tenenbaum, 2001; van Raalte et al., 1994). However, a limitation is that some behaviours mentioned by the coaches were not easily observable and therefore, were not added to the taxonomy; just to mention some examples: 'keeping fighting when exhausted' and 'no fun on the court'. Thus, although the taxonomy could be considered as a reliable and comprehensive instrument, it was not (yet) exhaustive.

Another limitation of the study is the number of participants. Therefore, any conclusions should be taken with caution. Only six tennis players participated in our research and just five of them played a three set match used for further analysis. However, this study should be considered as the first step into comparing different measures of mental toughness and using multi-source measures to get a more complete view of the mental toughness of talented youth tennis players. Further research should focus on expanding the number of participants to possibly draw better conclusions about the relationship between on-court observations and a self-reported measure of mental toughness in youth talented tennis players. An interesting avenue for future research would be also to examine whether players with different personality traits demonstrate different mentally tough behaviours on the court. The current results showed that some players expressed themselves much more on the court than other ones. It could be that these players were more extrovert and therefore, expressed themselves relatively more (Riggio and Riggio, 2002).

The results of this study could be used by coaches and sport psychologists to analyse and enhance the mental toughness of junior tennis players. First, coaches can use the developed taxonomy as a resource to observe junior tennis players during matches. In this way, these coaches might get a better view of their pupils' mental toughness. Second, coaches and sport psychologists could develop training programs to enhance mental toughness with the focus on lowering the variability of on-court behaviours during a match, thus, keeping a relatively stable 
emotional state.

In conclusion, in the current study a taxonomy was developed to investigate mentally tough behaviours and analysed how the observations were related to scores on a widely used questionnaire of mental toughness. Relating the on-court behaviours to the overall selfreported mental toughness, it seems that mentally tough behaviour is not reflected by the number of positive and negative behaviours, but by the variability of these behaviours during a match. Although research in sports sciences is often focused on average tendencies of athlete's behaviours or performance, we suggest that a focus on variability may reveal new insights into behaviours of athletes on the court.

Accordingly, coaches and sport psychologists should keep in mind that the key psychological skill for athletes may lie in their ability to keep a relatively stable state, which means they should not be too sensitive to the occurrences (or lack) of positive events during a match.

\section{Acknowledgements}

The authors would like to thank the coaches and junior tennis players who participated in this study, together with the Dutch Tennis Association (KNLTB) and the people who provided the opportunity to take recordings at several national junior ranking tournaments.

\section{References}

Bos J, Steenbeek H. MediaCoder: A simple application for coding behaviour within media files. Groningen, The Netherlands: University of Groningen; 2006

Bull SJ, Shambrook CJ, James W, Brooks JE. Towards an understanding of mental toughness in elite English cricketers. J Appl Sport Psychol, 2005; 17(3): 209-227

Clough PJ, Earle K, Sewell, D. Mental toughness: The concept and its measurement. In: Cockerill I, ed. Solutions in Sports Psychology. London: Thomson, 32-45; 2002

Clough PJ, Strycharczyk D. Develop mental toughness: Improving performance, wellbeing and positive behaviours in others. London: Kogan Page Limited; 2012

Cohen J. Statistical Power Analysis for the Behavioural Sciences, $2^{\text {nd }}$ Edition. Hillsdale: Lawrence Erlbaum; 1988

Crust L. A review and conceptual re-examination of mental toughness: Implications for future researchers. Pers Indiv Differ, 2008; 45(7): 576-583

Crust L. The relationship between mental toughness and affect intensity. Pers Indiv Differ, 2009; 47: 959-963

Diment GM. Mental toughness in soccer: A behavioral analysis. J Sport Behav, 2014; 37(4): 317

Elferink-Gemser M, Visscher C, Lemmink K, Mulder T. Relation between multidimensional performance characteristics and level of performance in talented youth field hockey players. J Sport Sci, 2004; 22(1112): 1053-1063

Gould D, Medbery R, Damarjian N, Lauer L. Survey of mental skills training knowledge, opinions and practices of junior tennis coaches. J Appl Sport Psychol, 1999; 11(1): 28-50

Gucciardi D, Gordon S, Dimmock J. Towards and understanding of mental toughness in Australian football. J Appl Sport Psychol, 2008; 20: 261-281

Hanegby R, Tenenbaum G. Blame it on the racket: norm-breaking behaviours among junior tennis players. Psychol Sport Exerc, 2001; 2(2): 117-134

Hartigh RJR den, Gernigon C, Van Yperen NW, Marin L, Van Geert PLC. How psychological and behavioral team states change during positive and negative momentum. PLoS ONE, 2014; 9(5): e97887

Hartigh RJR den, Van Geert PLC, Van Yperen NW, Cox RFA, Gernigon C. (2016). Psychological momentum 
during and across sports matches: evidence for interconnected time scales.J Sport Exercise Psy, 2016; 38: 82-92

Jones G, Hanton S, Connaughton D. What is this thing called mental toughness? An investigation of elite sport performers. J Appl Sport Psychol, 2002; 14: 205-218

Jones G, Hanton S, Connaughton D. A framework of mental toughness in the world's best performers. Sport Psychol, 2007; 21: 243-264

Kaiseler M, Polman R, Nicholls A. Mental toughness, stress, stress appraisal, coping and coping effectiveness in sport. Pers Indiv Differ, 2009; 47: 728-733

McHugh ML. Interrater reliability: the kappa statistic. Biochem Med, 2012; 22(3): 276-282

Middleton SC, Marsh HW, Martin, AJ, Richards GE, Savis J, Perry C. The Pyschological Performance Inventory: is the mental toughness test tough enough? Int J Sports Psychol, 2004; 35: 91-108

Nicholls AR, Perry JL, Jones L, Sanctuary C, Carson F, Clough PJ. The mediating role of mental toughness in sport. J Sport Med Phys Fit, 2015; 55(7-8): 824-834

Paulhus DL, Vazire S. The Self-Report Method. Handbook of research methods in personality psychology. New York: Guilford, 224-239; 2001

Perry JL, Clough PJ, Crust L, Earle K, Nicholls AR. Factorial validity of the mental toughness questionnaire48. Pers Indiv Differ, 2013; 54: 587-592

Raalte JL, van Brewer BW, Rivera PM, Petitpas AJ. The relationship between observable self-talk and competitive junior tennis players' match performance. J Sport Psychol, 1994; 16(4): 400-415

Riggio HR, Riggio RE. Emotional expressiveness, extraversion, and neuroticism: a meta-analysis. J Nonverbal Behav, 2002; 26(4): 195-218

Sheard M, Golby J. Effect of a psychological skills training program on swimming performance and positive psychological development. J Sport Exercise Psy, 2006a; 4: 149-169

Sheard M, Golby J. The efficacy of an outdoor adventure education curriculum on selected aspects of positive psychological development. J Exp Educ, 2006b; 29: 187-209

Strycharczyk D, Clough PJ. Developing mental toughness in young people: Approaches to achievement, well-being, employability and positive behaviour. London: Karnac Books; 2014

Weinberg R. The mental advantage: Developing your psychological skills in tennis. Champaign, IL: Human Kinetics; 1988

Weinberg R, Butt J, Culp B. Coaches' view of mental toughness and how it is built. Int J Sports Psychol, 2009; 9(2): 156-172

Yperen van NW. Why some make it and others do not: Identifying psychological factors that predict career success in professional adult soccer. Sport Psychol, 2009; 23: 317-329

\section{Corresponding author:}

Barbara Huijgen

Center for Human Movement Sciences, University Medical Center Groningen, University of Groningen.

Antonius Deusinglaan 1, 9713 AV Groningen, the Netherlands

Phone: +31/06/25646582

Fax: + 31/50/363 3150

E-mail: b.c.h.huijgen@umcg.nl 